\title{
Jetzt die eigenen Möglichkeiten für digitale Medizin prüfen und planen
}

\author{
Die Digitalisierung in der Medizin schreitet unaufhaltsam voran - \\ und das gilt nicht erst seit der Verabschiedung des E-Health- \\ Gesetzes Ende des vergangenen Jahres. Für niedergelassene \\ Fachärzte ist jetzt der Zeitpunkt gekommen, sich aktiv mit den \\ neuen Techniken zur Vernetzung sowohl mit Kollegen als auch \\ mit Patienten, mit Gesundheits-Apps und mit abgesicherter \\ Kommunikation zu beschäftigen.
}

Das E-Health-Gesetz ist vor allem ein Signal für die Heilberufler, sich auch in ihren Praxen auf digitale Prozesse vorzubereiten, dem könne man sich nicht mehr entziehen, so die Meinung von Georg Heßbrügge, Bereichsleiter Gesundheitsmärkte und -politik bei der Deutschen Apotheker- und Ärztebank (apoBank) im Gespräch mit Springer Medizin. Das gelte zum Beispiel auch für das ungeliebte Stammdatenmanagement der Krankenkassen, mit dem der aktuelle Versichertenstatus eines Patienten über die $\mathrm{Ge}$ sundheitskarte und die Telematikinfrastruktur automatisch online überprüft und bei Bedarf aktualisiert werden soll. „Das wird kommen“, prophezeit Heßbrügge. Die Tests dafür sollen nach mehrfacher Verzögerung noch in diesem Jahr beginnen, ab Juli 2018 sollen Ärzte dann verpflichtet sein, das in ihren Praxen $\mathrm{zu}$ machen.

\section{Die Fristen des E-Health-Gesetzes}

Die Fristen, die das E-Health-Gesetz vorgibt, so Heßbrügge, seien eine zweischneidige Sache für Fachärzte: „Einerseits ist das Tempo viel zu langsam, wenn man sieht, wie schnell die Digitalisierung in Deutschland vorankommt - mit der breiten Nutzung von Apps, von Smartphones und auch von sozialen Medien. Deswegen wird ja auch teilweise so hart Kritik daran geübt, dass zum Beispiel der Medikationsplan zunächst auf Papier kommen soll." Auf der anderen Seite seien die Fristen für die Selbstverwaltung durchaus anspruchsvoll, sei es bei der Umsetzung des Stammdatenmanagements, sei es bei neuen Leistungen wie dem Telekonsil und der Videosprechstunde, die bereits im kommenden Jahr wirksam werden sollen. Wohin die Reise geht, sehe man auch daran, dass die gematik laut Gesetz bis Ende dieses Jahres prüfen soll, inwieweit Patienten mit mobilen Geräten Zugriff auf ihre Patientendaten in einer künftigen elektronischen Patientenakte bekommen könnten.

\section{Nicht abwarten, bis die EBM-Ziffer kommt}

„Es ist an der Zeit, sich ganz konkret darüber Gedanken zu machen, wie die neuen Anwendungen umzusetzen sind“, mahnt Heßbrügge. Denn wenn Patienten sich an das System anschließen dürfen, könnte die elektronische Patientenakte, die bis Ende 2018 technisch bereitgestellt werden soll, sich sehr dynamisch entwickeln. Auch wenn die Unsicherheit noch groß ist, gehe es darum, die möglichen Anwendungen auf Realisierbarkeit in der eigenen Praxis zu prüfen, zum Beispiel zu überlegen: „Wie bette ich eine Videosprechstunde in die Praxisabläufe ein?" Vor allem sollten Ärzte mit der Umsetzung digitaler Prozesse nicht so lange warten, bis die Leistungen auch ausreichend honoriert werden. „Natürlich müssen digital erbrachte Leistungen auf die Dauer bezahlt werden. Aber darauf zu warten, welches Geld gezahlt werden wird, und erst dann zu investieren, wäre die falsche Haltung. Denn die Leistungen werden von immer mehr Patienten eingefordert", warnt Heßbrügge.

Nicht jede Fachgruppe bei den Ärzten müsse gleich schnell auf die Digitalisierung reagieren, räumt der Vordenker ein. „Eine Gynäkologin, die viele junge Mütter betreut, muss viel mehr damit rechnen, dass ihre Patientinnen erwarten, auch online Termine buchen zu können oder Befunde online abzurufen, als ein Orthopäde, der sich auf Reha-Patienten spezia-

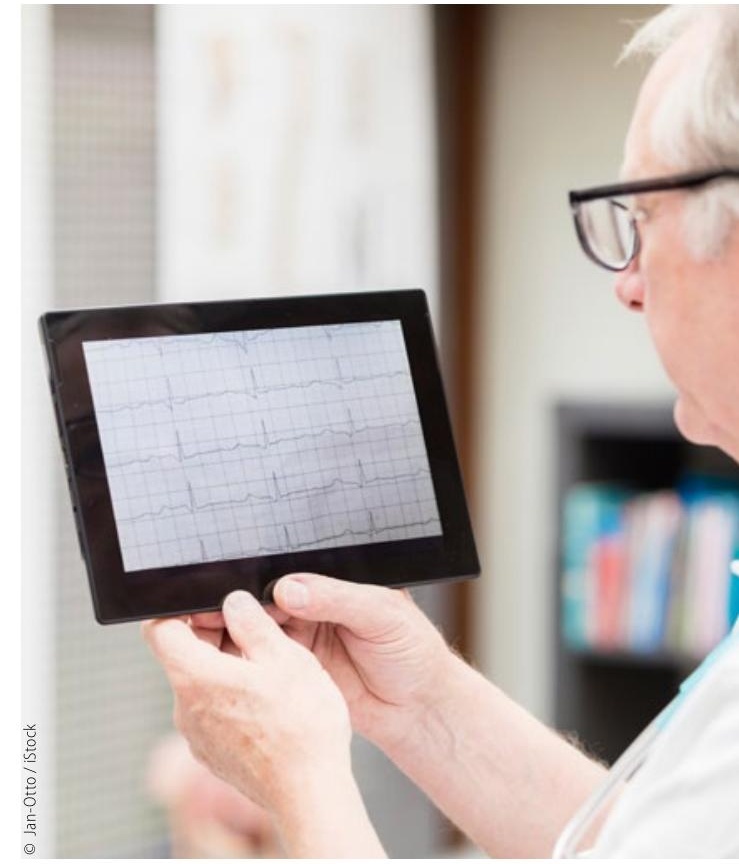

In Arztpraxen sind digitale Leistungen noch nicht der Alltag von heute, aber schon bald der von morgen. Der Patient wird sie einfordern, darauf sollten niedergelassene Ärzte vorbereitet sein.

lisiert hat." Bei Sportmedizinern sehe es natürlich ebenfalls anders aus als bei Geriatern - wobei die auch schon wieder über den Kontakt mit den betreuenden Angehörigen oder auch mit Pflegeheimen Nachfragen nach digitalen Anwendungen gegenüberstehen und mit Kompetenz in Telemedizin punkten könnten.

Ein Arzt, der sich der neuen Technik eher langsam nähert, verliere sicher nicht auf einmal alle Patienten, aber es könne doch ein schleichender Prozess einsetzen, der verhindert, dass neue Patienten für die Praxis gewonnen werden. Wenn der Arzt keine Online-Terminbuchungen ermögliche, führt das bereits in manchen Patientenkreisen zu der Vermutung, "dass dann auch das Ultraschallgerät in der Praxis wohl schon etwas älter ist. Da passiert etwas, das für Ärzte hoch relevant ist, ohne dass im EBM Ziffern dafür gebaut sind“, warnt Heßbrügge.

Hauke Gerlof 